\title{
Evaluar los conocimientos sobre el testamento vital, de enfermeros y pacientes con IRCT, en los servicios de hemodiálisis del hospital Joan xxIII de Tarragona y del hospital Pius de Valls
}

\author{
Susana Abejaro Rodríguez, Yolanda Benito García, Natàlia Veciana Magriña, Mercedes Prats Valencia, \\ Antonio Marca Gutiérrez
}

Hospital Universitari Joan XXIII. Tarragona

\section{Introducción:}

Como profesionales del servicio de Diálisis de un Hospital de referencia provincial, nos encontramos con gran variedad de pacientes, algunos crónicos y otros agudos. En muchas ocasiones, creemos que sería necesario respetar el proceso natural del fin de la vida, de una manera digna. Pero no siempre es fácil decidir. Nos preguntamos si toda la tecnología empleada estará resultando fútil. Incluso, hay situaciones en las que el propio paciente pide que se le retire de la diálisis. Nuestra experiencia como personal de hemodiálisis nos induce a pensar que todo resultaría más fácil si los pacientes con Insuficiencia Renal Crónica Terminal (IRCT) pudieran elaborar su propio documento de voluntades anticipadas. Nuestro estudio se basa en conocer la información que tiene el profesional sanitario y pacientes con IRCT de las unidades de hemodiálisis del Hospital $X$ y del Hospital $Y$ sobre el documento de voluntades anticipadas ( DVA). Este proyecto pertenece al paradigma positivista, es un estudio observacional, de tipo transversal, descriptivo y correlacional. La población estudio son los pacientes con IRCT que se dializan en los centros mencionados y los profesionales que trabajan en dichas unidades de hemodiálisis. Los datos se recogen durante un mes con una muestra de 50 pacientes y 30 profesionales. Se encuesta a toda la población seleccionada. Las variables a estudiar son: la información que poseen el personal y los pacientes sobre el DVA. También se realiza un perfil del paciente con IRCT en hemodiálisis, por tanto, se estudia también las variables sociodemográficas 0 atributivas. Para la recogida de datos se utilizarán dos cuestionarios. En primer lugar se encuestarán a los pacientes con una encuesta validada, elaborada por el grupo de Ética y Nefrología de la Sociedad Española de Nefrología (SEN),
2011. Los pacientes recibirán una documentación explicativa sobre la naturaleza y el significado de las DVA y un cuestionario sobre su conocimiento y su aceptación. En segundo lugar se encuestarán al personal con un cuestionario validado que consta de 12 ítems. Este cuestionario explora las variables sociodemográficas (edad, sexo y nivel asistencial) además de los conocimientos y actitudes de estos profesionales sanitarios acerca de las DVA. En el análisis de datos univariante se utilizarán tablas de frecuencias y porcentajes para las variables categóricas y medidas de centralización y dispersión para las variables cuantitativas. En el análisis Bivariante o Multivariante se utilizará el análisis de regresión. El programa estadístico utilizado será SPSS 15.0. Las palabras claves utilizadas serán: Cuidados del fin de la vida. Voluntades Anticipadas. Planificación anticipada de cuidados Enfermedad Renal Crónica. Paciente con IRCT. Enfermera de Hemodiálisis.

\section{Referencias Bibliográficas}

1. Antolín A, et al. Evolución temporal en el conocimiento y el posicionamiento de los pacientes con enfermedades crónicas respecto al testamento vital. Gac Sanit. 2001.doi:10.1016/j.gaceta.2011.03.009.

2. Sánchez Tomero JA.. Planificación anticipada e inicio de diálisis. Nefrología 2009;29:285-287.

3. Rodríguez Jornet A, Ibeas J, Real J, Peña S, Martínez Ocaña JC, García García M. Documento de voluntades anticipadas de pacientes con insuficiencia renal crónica terminal en tratamiento sustitutivo mediante diálisis. Nefrología 2007;27:581-592. 
4. Sánchez Tomero JA, Rodríguez Jornet $A$, Balda $S$, Cigarrán S, Herero JC, Maduell F, et al. Evaluación de la opinión de los pacientes con enfermedad renal crónica en diálisis respecto al fin de la vida y la planificación anticipada de cuidados. Nefrología. 2011; 31: 449-456.

5. Mendoza del Solar G. El concepto de futilidad en la práctica médica. Soc Peru Med Interna. 2008; 21:26-35.

6. Díaz Jurado M, Simal Vélez N, Salillas Adot E, Julve Ibáñez M. Aproximaciones al principio de autonomía en el tratamiento de diálisis: el consentimiento informado y las voluntades anticipadas. Soc Esp Enfer Nefrol. 2009; 12 (3): 184-188.

7. Fernández Viña-Viña Fernández AM, González Suárez E, Díaz Vigil-Escalera P, Reguera Álvarez R,
Nieto García JM, Naves Díaz M. La actitud ante la muerte en el paciente renal en programa de hemodiálisis, a través de encuesta sobre el conocimiento del testamento vital. Seden. [Revista on line][Consultado el 10 de marzo de 2013] Disponible en: http://www.revistaseden.org/files/2848_130-132. pdf Rodríguez Jornet A. Acerca de la retirada de diálisis.Nefrología. 2001; 21 (5): 433-434.

8. Palero Castello C. Documento de instrucciones previas y afrontación del proceso de morir en el enfermo renal crónico terminal en hemodiálisis. Perspectiva antropológica. Seden. [Revista on line][Consultado el 12 de marzo de 2013] Disponible en: http://www.revistaseden.org/ files/2127_P\%C3\%Alginas\%20de\%202009-12. pdf Mattiussi M. Perspectivas de los pacientes sobre las voluntades anticipadas y tratamientos en la etapa terminal. Isalud. 2009; 8: 21. 